PROCEEDINGS OF THE

AMERICAN MATHEMATICAL SOCIETY

Volume 131, Number 5, Pages 1459-1469

S 0002-9939(02)06782-5

Article electronically published on September 19, 2002

\title{
ON POINTWISE ESTIMATES FOR THE LITTLEWOOD-PALEY OPERATORS
}

\author{
ANDREI K. LERNER
}

(Communicated by Andreas Seeger)

\begin{abstract}
In a recent paper we proved pointwise estimates relating some classical maximal and singular integral operators. Here we show that inequalities essentially of the same type hold for the Littlewood-Paley operators.
\end{abstract}

\section{INTRODUCTION}

Let $\omega$ be a non-negative, locally integrable function on $\mathbb{R}^{n}$. Given a measurable set $E$, let $\omega(E)=\int_{E} \omega(x) d x$. The non-increasing rearrangement of a measurable function $f$ on $\mathbb{R}^{n}$ with respect to $\omega$ is defined by

$$
f_{\omega}^{*}(t)=\sup _{\omega(E)=t} \inf _{x \in E}|f(x)| \quad(0<t<\infty) .
$$

If $\omega \equiv 1$ we use the notation $f^{*}(t)$.

Let us consider the maximal function (cf. [13])

$$
m_{\lambda} f(x)=\sup _{Q \ni x}\left(f \chi_{Q}\right)^{*}(\lambda|Q|) \quad(0<\lambda<1),
$$

where the supremum is taken over all cubes $Q$ containing $x, \chi_{Q}$ denotes the indicator function of $Q$ and $|Q|$ denotes the Lebesgue measure of $Q$.

The function $m_{\lambda} f$, as well as the Hardy-Littlewood maximal function $M f$, is a pointwise majorant of $|f|$. However, $m_{\lambda} f$ and $f$ have the same integrability properties in the $L^{p}$ scale for any $p>0$ (this follows, for example, from inequality (3.1) below), unlike $M f$ for which it is true only for $p>1$.

In [9, the following pointwise estimate was proved:

$$
m_{\lambda}\left(T_{1} f\right)(x) \leq c_{\lambda, n} T_{2} f(x)+T_{1} f(x)
$$

where $T_{1}, T_{2}$ are certain maximal and singular integral operators. More precisely, (1.1) was proved in the cases when either $T_{1}$ or $T_{2}$ is the Hardy-Littlewood maximal function, while $T_{2}$ or $T_{1}$ is the Fefferman-Stein sharp maximal function and the Calderón-Zygmund maximal singular integral operator, respectively. Such estimates easily imply weighted rearrangement and $L^{p}$-inequalities with respect to a weight satisfying the $A_{\infty}$ condition; $B L O$-inequalities can be derived as well (see Lemma 1 in [9] for further details). Thus they allow us to unify and simplify some basic techniques extensively applied to the above-mentioned operators.

Received by the editors December 7, 2001.

2000 Mathematics Subject Classification. Primary 42B25.

Key words and phrases. Littlewood-Paley operators, pointwise estimates, rearrangements.

(C)2002 American Mathematical Society 
The aim of this paper is to show that estimates essentially of the same type as (1.1) hold for the Littlewood-Paley operators. The role of these operators in harmonic analysis is well-known (see, e.g., [12] Ch. 4]). Among numerous papers on this subject, let us mention only those of specific interest to us, namely those where "good- $\lambda$ " [1, 4, 5, 10, 11, rearrangement 7] and BLO [8] inequalities were proved.

Roughly speaking, our central result states that for certain Littlewood-Paley operators $T_{1}$ and $T_{2}$ we have the pointwise estimates of the form

$$
m_{\lambda}\left(T_{1} f\right)(x) \leq c_{\lambda, n} m_{\lambda / 4}\left(T_{2} f\right)(x)+T_{1} f(x) .
$$

Below, in Lemma 3.5, we show that such estimates imply the results of no less importance than (1.1) implies.

Two main approaches to the study of the Littlewood-Paley operators are wellknown. The first one is based on the vector-valued analysis, while the second is based on the theory of harmonic functions. We shall use the latter approach, since it is not quite clear how one can combine the rearrangement and vector-valued techniques.

The paper is organized as follows. Section 2 contains some preliminaries. In Section 3, we state the main results, Theorems 3.1 3.4 and show how to apply them. In Section 4, we state and prove two lemmas which form the core of the proofs of Theorems 3.1 and 3.2. In Section 5, we prove Theorems 3.1 3.4

\section{Preliminaries}

Throughout the paper, $u$ will be a harmonic function on the upper half space $\mathbb{R}_{+}^{n+1}=\mathbb{R}^{n} \times \mathbb{R}_{+}$.

Define the truncated Lusin area integral and nontangential maximal function by

$$
S_{\alpha, h} u(x)=\left(\int_{\Gamma_{\alpha, h}(x)} t^{1-n}|\nabla u(y, t)|^{2} d y d t\right)^{1 / 2}
$$

and

$$
N_{\alpha, h} u(x)=\sup _{(y, t) \in \Gamma_{\alpha, h}(x)}|u(y, t)|,
$$

respectively, where $\nabla u=\left(\frac{\partial u}{\partial x_{1}}, \ldots, \frac{\partial u}{\partial x_{n}}, \frac{\partial u}{\partial t}\right)$ and $\Gamma_{\alpha, h}$ is the truncated cone with vertex at $x \in \mathbb{R}^{n}$ and aperture $\alpha$ :

$$
\Gamma_{\alpha, h}(x)=\left\{(y, t) \in \mathbb{R}_{+}^{n+1}:|y-x|<\alpha t, 0<t<h\right\} .
$$

When $h=+\infty$ we drop the subscript $h$ and write $S_{\alpha}, N_{\alpha}, \Gamma_{\alpha}$. Also we consider the Littlewood-Paley functions $g$ and $g_{\mu}$ defined by

$$
g(u)(x)=\left(\int_{0}^{\infty} t|\nabla u(x, t)|^{2} d t\right)^{1 / 2}
$$

and

$$
g_{\mu}(u)(x)=\left(\int_{\mathbb{R}_{+}^{n+1}}\left(\frac{t}{t+|x-y|}\right)^{\mu n} t^{1-n}|\nabla u(y, t)|^{2} d y d t\right)^{1 / 2} \quad(\mu>1) .
$$

Next, we define the vertical and box maximal functions by

$$
N^{+} u(x)=\sup _{t>0}|u(x, t)|
$$


and

$$
T_{\mu} u(x)=\sup _{Q \ni x}\left(\frac{1}{|Q|^{\mu}} \int_{T(Q)} t^{(\mu-1) n-1}|u(y, t)|^{2} d y d t\right)^{1 / 2} \quad(\mu>1),
$$

respectively, where $Q$ is a cube, $T(Q)=\left\{(x, t): x \in Q, 0<t<d_{Q}\right\}$, and $d_{Q}$ denotes the diameter of $Q$.

Following Strömberg [13, define the local square function by

$$
S_{\lambda, \alpha}^{\#} u(x)=\sup _{Q \ni x}\left(\left(S_{\alpha, d_{Q}} u\right) \chi_{Q}\right)^{*}(\lambda|Q|) \quad(0<\lambda<1) .
$$

Consider the "local nontangential" maximal function defined by

$$
N_{\lambda, \alpha}^{\#} u(x)=\sup _{Q \ni x} \inf _{c \in \mathbb{R}}\left(\left(N_{\alpha, d_{Q}}(u-c)\right) \chi_{Q}\right)^{*}(\lambda|Q|) \quad(0<\lambda<1) .
$$

It is easy to see that $S_{\lambda, \alpha}^{\#} u(x) \leq m_{\lambda} S_{\alpha} u(x)$ and $N_{\lambda, \alpha}^{\#} u(x) \leq m_{\lambda} N_{\alpha} u(x)$.

Now, we recall some well-known facts concerning harmonic functions. First, by Green's Theorem,

$$
\int_{G} t|\nabla u(y, t)|^{2} d y d t=\int_{\partial G}\left(t u \frac{\partial u}{\partial n}-\frac{u^{2}}{2} \frac{\partial t}{\partial n}\right) d \sigma,
$$

where $G$ is a domain in $\mathbb{R}_{+}^{n+1}$ with piecewise smooth boundary $\partial G$. Further, by mean-value property we have for $\alpha<\beta, h<h^{\prime}$ (see [12, p. 207]),

$$
\sup _{(y, t) \in \Gamma_{\alpha, h}(x)} t|\nabla u(y, t)| \leq c N_{\beta, h^{\prime}} u(x)
$$

and

$$
\sup _{(y, t) \in \Gamma_{\alpha, h}(x)} t|\nabla u(y, t)| \leq c S_{\beta, h^{\prime}} u(x)
$$

where $c=c\left(\alpha, \beta, h, h^{\prime}, n\right)$.

Recall that a weight $\omega$ satisfies Muckenhoupt's condition $A_{\infty}$ if there exist $c, \delta>0$ such that for any $Q$ and $E \subset Q$,

$$
\omega(E) \leq c(|E| /|Q|)^{\delta} \omega(Q) .
$$

The space $B L O$ [3] consists of all functions $f \in L_{l o c}^{1}\left(\mathbb{R}^{n}\right)$ such that

$$
\|f\|_{B L O}=\sup _{Q} \frac{1}{|Q|} \int_{Q}\left(f(x)-\inf _{Q} f\right) d x<\infty .
$$

\section{Main Results AND their applications}

Our main results are the following.

Theorem 3.1. For any harmonic function $u$ on $\mathbb{R}_{+}^{n+1}$ and for all $x \in \mathbb{R}^{n}$,

$$
m_{\lambda}\left(S_{\alpha}^{2} u\right)(x) \leq c m_{\lambda / 4}\left(N_{\beta}^{2} u\right)(x)+S_{\alpha}^{2} u(x) \quad(0<\lambda<1, \alpha<\beta),
$$

where $c$ is a constant depending on $\lambda, \alpha, \beta$ and $n$.

Theorem 3.2. For any harmonic function $u$ on $\mathbb{R}_{+}^{n+1}$ and for all $x \in \mathbb{R}^{n}$,

$$
m_{\lambda}\left(N_{\alpha} u\right)(x) \leq c m_{\lambda / 4}\left(S_{\beta} u\right)(x)+N^{+} u(x) \quad(0<\lambda<1, \alpha<\beta),
$$

where $c$ is a constant depending on $\lambda, \alpha, \beta$ and $n$. 
Theorem 3.3. For any harmonic function $u$ on $\mathbb{R}_{+}^{n+1}$ and for all $x \in \mathbb{R}^{n}$,

$$
m_{\lambda}\left(g^{2} u\right)(x) \leq c m_{\lambda / 4}\left(N_{\alpha}^{2} u\right)(x)+g^{2} u(x) \quad(0<\lambda<1, \alpha>0),
$$

where $c$ is a constant depending on $\lambda, \alpha$ and $n$.

Theorem 3.4. For any harmonic function $u$ on $\mathbb{R}_{+}^{n+1}$ and for all $x \in \mathbb{R}^{n}$,

$$
m_{\lambda}\left(g_{\mu}^{2}(u)\right)(x) \leq c m_{\lambda / 4}\left(T_{\mu}^{2} u\right)(x)+g_{\mu}^{2} u(x) \quad(0<\lambda<1, \mu>1),
$$

where $c$ is a constant depending on $\lambda, \mu$ and $n$.

The following result clarifies the sense of (1.2), or more precisely of Theorems 3.1-3.4. As its counterpart for (1.1), Lemma 1 in [9], it serves as a bridge between a certain relation for two functions and corresponding relations for their rearrangements and norms.

Lemma 3.5. For any $\lambda, 0<\lambda \leq 1 / 2$, let there exist a constant $c_{\lambda}>0$, so that the non-negative functions $f, g$ satisfy the inequality

$$
m_{\lambda} f(x) \leq c_{\lambda} m_{\lambda / 4} g(x)+f(x)
$$

for all $x \in \mathbb{R}^{n}$, and let $\omega \in A_{\infty}$. Then we have:

(i) there exist constants $c_{1}, c_{2}>0$, so that for all $t>0$,

$$
f_{\omega}^{*}(t) \leq c_{1} g_{\omega}^{*}\left(c_{2} t\right)+f_{\omega}^{*}(2 t)
$$

(ii) if $f_{\omega}^{*}(+\infty)=0$, then

$$
\|f\|_{L_{\omega}^{p}} \leq c_{p}\|g\|_{L_{\omega}^{p}} \quad(0<p<\infty)
$$

(iii) if $g \in L^{\infty}$, then $f$ has bounded lower oscillation, $f \in B L O$, and

$$
\|f\|_{B L O} \leq c\|g\|_{\infty} .
$$

Since the proof goes along the same lines as Lemma 1 in [9], we outline it briefly. Actually, all items of this lemma are an immediate combination of Lemma 1 in [9] and the next inequality

$$
\left(m_{\lambda} f\right)_{\omega}^{*}(t) \leq f_{\omega}^{*}(c t),
$$

where $c=c(\lambda, \omega)$. To prove (3.1), observe that from the definition of the rearrangement we have

$$
\left\{x: m_{\lambda} f(x)>\alpha\right\} \subset\left\{x: M \chi_{\{y:|f(y)|>\alpha\}}(x) \geq \lambda\right\} \quad(\lambda, \alpha>0) .
$$

On the other hand, it is well known (see, e.g., 2] ) that a weight $\omega$ belongs to $A_{\infty}$ iff there exist $k, r \geq 1$ so that

$$
\omega\{x: M f(x)>\lambda\} \leq \frac{k}{\lambda^{r}} \int_{\mathbb{R}^{n}}|f(x)|^{r} \omega(x) d x
$$

for all measurable functions $f$ on $\mathbb{R}^{n}$ and all $\lambda>0$. Using these inequalities, we obtain

$$
\omega\left\{x: m_{\lambda} f(x)>\alpha\right\} \leq c \omega\{x:|f(x)|>\alpha\},
$$

which proves (3.1).

Remark 3.6. It is easy to see that all assertions of Lemma 3.5 hold under the assumption that non-negative functions $f, g$ satisfy the inequality

$$
m_{\lambda} f(x) \leq c_{\lambda} m_{\varphi(\lambda)} g(x)+f(x),
$$

where $\varphi(\lambda)$ is a measurable function satisfying the only condition $0<\varphi(\lambda)<1$. 
Theorems 3.13 .4 together with (ii) of Lemma 3.5 give classical $L_{\omega}^{p}$-estimates obtained earlier with the help of "good- $\lambda$ " inequalities. Also, using Theorems 3.1$]$ [3.3, 3.4 and (iii) of Lemma 3.5] we get a new approach to $B L O$ inequalities (cf. [8]).

Next we note that Theorems 3.1, 3.3, 3.4 and (i) of Lemma 3.5 give a refinement of the rearrangement inequalities proved in [7].

Finally, we would like to point out that combining Theorem 3.2 and (i), (iii) of Lemma 3.5 gives two new results concerning the nontangential maximal function.

Corollary 3.7. Let $\omega \in A_{\infty}$. For any harmonic function $u$ on $\mathbb{R}_{+}^{n+1}$ we have:

(i) there exist constants $c^{\prime}, c^{\prime \prime}>0$ depending on $\alpha, \beta, \omega$, so that

$$
\left(N_{\alpha} u\right)_{\omega}^{*}(t) \leq c^{\prime}\left(S_{\beta} u\right)_{\omega}^{*}\left(c^{\prime \prime} t\right)+\left(N_{\alpha} u\right)_{\omega}^{*}(2 t) \quad(t>0, \alpha<\beta) ;
$$

(ii) if $S_{\beta} u \in L^{\infty}$, then $N_{\alpha} u \in B L O$, and

$$
\left\|N_{\alpha} u\right\|_{B L O} \leq c\left\|S_{\beta} u\right\|_{\infty} \quad(\alpha<\beta) .
$$

\section{Two Lemmas}

We shall establish pointwise relations between $S_{\lambda, \alpha}^{\#} u$ and $N_{\lambda, \alpha}^{\#} u$. This is a key ingredient in the proving Theorems 3.1 and 3.2 . Note that we partially use the arguments from [1, 5, 11.

Lemma 4.1. For all $x \in \mathbb{R}^{n}$,

$$
S_{\lambda, \alpha}^{\#} u(x) \leq c N_{\lambda^{\prime}, \beta}^{\#} u(x) \quad\left(0<\lambda^{\prime}<\lambda<1, \alpha<\beta\right),
$$

where $c=c\left(\lambda, \lambda^{\prime}, \alpha, \beta, n\right)$.

Proof. Let $x \in Q$. For $\delta>0$ let $h=(1+\delta) d_{Q}$, and

$$
A=\left\{z \in Q: N_{\beta, h} u(z) \leq\left(\left(N_{\beta, h} u\right) \chi_{Q}\right)^{*}((\lambda-\delta)|Q|)\right\} .
$$

It is clear that $|A| \geq(1-\lambda+\delta)|Q|$. Let $E \subset Q$ be an arbitrary set with $|E|=\lambda|Q|$. Then $|E \cap A| \geq \delta|Q|$.

Set $D=\bigcup_{\xi \in E \cap A} \Gamma_{\alpha, d_{Q}}(\xi)$. Fubini's theorem yields

$$
\begin{aligned}
\inf _{z \in E} S_{\alpha, d_{Q}}^{2} u(z) & \leq \frac{1}{|E \cap A|} \int_{E \cap A} S_{\alpha, d_{Q}}^{2} u(z) d z \\
& \leq \frac{1}{\delta|Q|} \int_{D} t^{1-n}|\nabla u(y, t)|^{2}\left|\left\{\xi \in E \cap A:(y, t) \in \Gamma_{\alpha, d_{Q}}(\xi)\right\}\right| d y d t \\
& \leq \frac{c}{|Q|} \int_{D} t|\nabla u(y, t)|^{2} d y d t .
\end{aligned}
$$

Now one can estimate the last integral in a standard way. We approximate $D$ by a family of subdomains $D_{\varepsilon} \subset D$ with sufficiently smooth boundaries (see [12. p. 206]). It is easy to see that $\sigma\left(D_{\varepsilon}\right) \leq c|Q|$. Applying (2.1) and (2.2), we get

$$
\begin{aligned}
\int_{D_{\varepsilon}} t|\nabla u(y, t)|^{2} d y d t & \leq \int_{\partial D_{\varepsilon}}\left(t|u(y, t)||\nabla u(y, t)|+|u(y, t)|^{2} / 2\right) d \sigma \\
& \leq c \sigma\left(\partial D_{\varepsilon}\right) \sup _{z \in A} N_{\beta, h}^{2} u(z) \leq c|Q|\left(\left(N_{\beta, h}^{2} u\right) \chi_{Q}\right)^{*}((\lambda-\delta)|Q|) .
\end{aligned}
$$

Letting $D_{\varepsilon} \rightarrow D$ as $\varepsilon \rightarrow 0+$ and using (4.2), we have

$$
\inf _{z \in E} S_{\alpha, d_{Q}} u(z) \leq c\left(\left(N_{\beta, h} u\right) \chi_{Q}\right)^{*}((\lambda-\delta)|Q|) .
$$


Taking the supremum over all sets $E \subset Q$ with $|E|=\lambda|Q|$ yields

$$
\begin{aligned}
\left(\left(S_{\alpha, d_{Q}} u\right) \chi_{Q}\right)^{*}(\lambda|Q|) & \leq c\left(\left(N_{\beta, h} u\right) \chi_{Q}\right)^{*}((\lambda-\delta)|Q|) \\
& \leq c\left(\left(N_{\beta, h} u\right) \chi_{(1+\delta) Q}\right)^{*}\left(\lambda^{\prime}|(1+\delta) Q|\right),
\end{aligned}
$$

where $\lambda^{\prime}=(\lambda-\delta) /(1+\delta)^{n}$. This completes the proof, since $S_{\alpha, d_{Q}}(u-c)=S_{\alpha, d_{Q}}(u)$.

Now we introduce the following notation. Let $\alpha^{\prime}=\max (1 / \alpha, 1)$. If $x \in Q$, we set

$$
\tilde{u}(y, t)=u(y, t)-u\left(x, \alpha^{\prime} d_{Q}\right) .
$$

Lemma 4.2. For any cube $Q \subset \mathbb{R}^{n}$ and all $x \in Q$,

$$
\left(\left(N_{\alpha, d_{Q}} \tilde{u}\right) \chi_{Q}\right)^{*}(\lambda|Q|) \leq c m_{\lambda / 2} S_{\beta} u(x) \quad(0<\lambda<1, \alpha<\beta),
$$

where $c=c(\lambda, \alpha, \beta, n)$.

Proof. Let $x \in Q$. For $\delta>0$ let $h=(1+\delta) \alpha^{\prime} d_{Q}, S_{\beta}(Q)=\left(\left(S_{\beta, h} u\right) \chi_{Q}\right)^{*}((\lambda-\delta)|Q|)$ and

$$
A=\left\{z \in Q: S_{\beta, h} u(z) \leq S_{\beta}(Q)\right\} .
$$

Let $E \subset Q$ be an arbitrary set with $|E|=\lambda|Q|$. Then $|E \cap A| \geq \delta|Q|$.

For any $z \in E \cap A$ there exists a point $(y, t) \in \Gamma_{\alpha, d_{Q}}(z)$ so that $N_{\alpha, d_{Q}} \tilde{u}(z) \leq$ $2|\tilde{u}(y, t)|$. Let $\xi \in B(z, \alpha t)$, where $B(z, \alpha t)$ is the ball centered at $z$ of radius $\alpha t$. Since $(\xi, t),(y, t) \in \Gamma_{\alpha, d_{Q}}(z)$, all points of the segment with the end points $(\xi, t)$ and $(y, t)$ belong to $\Gamma_{\alpha, d_{Q}}(z)$. Using Mean Value Theorem, (2.3), and the fact that $\nabla \tilde{u}=\nabla u$, we obtain

$$
|\tilde{u}(y, t)| \leq c S_{\beta}(Q)+\inf _{\xi \in B(z, \alpha t)}|\tilde{u}(\xi, t)| .
$$

The balls $B(z, 2 \alpha t)$ form the covering of the set $E \cap A$. Using Besicovitch's theorem [6, p. 5], choose a finite number of pairwise disjoint balls $B\left(z_{i}, 2 \alpha t_{i}\right)(i=$ $1, \ldots, k)$ so that $\sum_{i=1}^{k}\left|B\left(z_{i}, \alpha t_{i}\right)\right| \geq c|E \cap A| \geq c^{\prime}|Q|$. Then

$$
\begin{aligned}
\inf _{z \in E} N_{\alpha, d_{Q}} \tilde{u}(z) & \leq \min _{1 \leq i \leq k} N_{\alpha, d_{Q}} \tilde{u}\left(z_{i}\right) \\
& \leq c\left(S_{\beta}(Q)+\min _{1 \leq i \leq k} \inf _{\xi \in B\left(z_{i}, \alpha t_{i}\right)}\left|\tilde{u}\left(\xi, t_{i}\right)\right|\right) .
\end{aligned}
$$

Now set $D=\bigcup_{i=1}^{k} \Gamma_{\alpha, d_{Q}}\left(z_{i}\right) \backslash \Gamma_{\alpha, t_{i}}\left(z_{i}\right)$. Let us denote $\partial D^{+}=\left\{\left(y, d_{Q}\right) \in \partial D\right\}$ and $\partial D^{-}=\partial D \backslash \partial D^{+}$. Since $B\left(z_{i}, 2 \alpha t_{i}\right)$ are disjoint and $\xi \notin B(z, 2 \alpha t)$ implies $\Gamma_{\alpha, t}(\xi) \cap \Gamma_{\alpha, t}(z)=\emptyset$, we see that $\bigcup_{i=1}^{k}\left\{\left(\xi, t_{i}\right): \xi \in B\left(z_{i}, \alpha t_{i}\right)\right\} \subset \partial D^{-}$. Therefore,

$$
\begin{aligned}
\min _{1 \leq i \leq k} \inf _{\xi \in B\left(z_{i}, \alpha t_{i}\right)}\left|\tilde{u}\left(\xi, t_{i}\right)\right| & \leq \min _{1 \leq i \leq k}\left(\frac{1}{\left|B\left(z_{i}, \alpha t_{i}\right)\right|} \int_{B\left(z_{i}, \alpha t_{i}\right)} \tilde{u}^{2}\left(\xi, t_{i}\right) d \xi\right)^{1 / 2} \\
& \leq\left(\frac{1}{\sum_{i=1}^{k}\left|B\left(z_{i}, \alpha t_{i}\right)\right|} \sum_{i=1}^{k} \int_{B\left(z_{i}, \alpha t_{i}\right)} \tilde{u}^{2}\left(\xi, t_{i}\right) d \xi\right)^{1 / 2} \\
& \leq\left(\frac{c}{|Q|} \int_{\partial D^{-}} \tilde{u}^{2} d \sigma\right)^{1 / 2} .
\end{aligned}
$$

It is easy to see that $|\partial t / \partial n| \leq 1, \sigma(\partial D) \leq c|Q|$ and $-\partial t / \partial n \geq c>0$ for $(y, t) \in \partial D^{-}$. Thus, by (2.1) we obtain

$$
\int_{\partial D^{-}} \tilde{u}^{2} d \sigma \leq c\left(\int_{\partial D^{+}} \tilde{u}^{2} d \sigma+\int_{\partial D} t|\tilde{u}||\nabla u| d \sigma+\int_{D} t|\nabla u(y, t)|^{2} d y d t\right) .
$$


Let $\left(y, d_{Q}\right) \in \partial D$. There exists $z_{i} \in A$ so that $\left|y-z_{i}\right|<\alpha d_{Q}$. Besides, $\left|x-z_{i}\right|<$ $d_{Q} \leq \alpha \cdot \alpha^{\prime} d_{Q}$. Since $\left(y, d_{Q}\right)$ and $\left(x, \alpha^{\prime} d_{Q}\right)$ belong to $\Gamma_{\alpha}\left(z_{i}\right)$, using Mean Value Theorem and (2.3), we have

$$
\left|\tilde{u}\left(y, d_{Q}\right)\right| \leq c S_{\beta, h} u\left(z_{i}\right) \leq c S_{\beta}(Q) .
$$

Hence,

$$
\int_{\partial D^{+}} \tilde{u}^{2} d \sigma \leq c|Q| S_{\beta}^{2}(Q)
$$

Next, by (2.3) and Hölder's inequality,

$$
\begin{aligned}
\int_{\partial D} t|\tilde{u}||\nabla u| d \sigma & =\int_{\partial D^{+}} t|\tilde{u}||\nabla u| d \sigma+\int_{\partial D^{-}} t|\tilde{u}||\nabla u| d \sigma \\
& \leq c|Q| S_{\beta}^{2}(Q)+\left(\int_{\partial D^{-}}(t|\nabla u|)^{2} d \sigma\right)^{1 / 2}\left(\int_{\partial D^{-}} \tilde{u}^{2} d \sigma\right)^{1 / 2} \\
& \leq c|Q| S_{\beta}^{2}(Q)+c|Q|^{1 / 2} S_{\beta}(Q)\left(\int_{\partial D^{-}} \tilde{u}^{2} d \sigma\right)^{1 / 2} .
\end{aligned}
$$

Let us estimate the last term in (4.5). We invoke the following geometrical observation (see [5]): for any point $\xi \in \mathbb{R}^{n}$ there exists $z \in A$ so that

$$
\left(\bigcup_{\eta \in A} \Gamma_{\alpha, d_{Q}}(\eta)\right) \cap \Gamma_{(\beta-\alpha) / 2, d_{Q}}(\xi) \subset \Gamma_{\beta, d_{Q}}(z) .
$$

It follows from this that for all $\xi \in \mathbb{R}^{n}$,

$$
\int_{\Gamma_{\frac{\beta-\alpha}{2}, d_{Q}(\xi)}} t^{1-n}|\nabla u(y, t)|^{2} \chi_{\eta \in A} \Gamma_{\Gamma_{\alpha, d_{Q}}(\eta)}(y, t) d y d t \leq S_{\beta}^{2}(Q) .
$$

Note that $\bigcup_{\eta \in A} \Gamma_{\alpha, d_{Q}}(\eta) \subset c^{\prime} Q \times d_{Q} \subset \bigcup_{\xi \in c^{\prime} Q} \Gamma_{(\beta-\alpha) / 2, d_{Q}}(\xi)$, with $c^{\prime}=2 \alpha \sqrt{n}+1$. Integrating (4.8) over $c^{\prime} Q$ and using Fubini's theorem, we get

$$
\begin{aligned}
& \int_{D} t|\nabla u(y, t)|^{2} d y d t \leq \int_{\eta \in A} \int_{\Gamma_{\alpha, d_{Q}}(\eta)} t|\nabla u(y, t)|^{2} d y d t \\
\leq & c \int_{\cup_{\eta \in A} \Gamma_{\alpha, d_{Q}}(\eta)} t^{1-n}|\nabla u(y, t)|^{2}\left|\left\{x^{\prime} \in c^{\prime} Q:(y, t) \in \Gamma_{\frac{\beta-\alpha}{2}, d_{Q}}\left(x^{\prime}\right)\right\}\right| d y d t \\
\leq & c\left|c^{\prime} Q\right| S_{\beta}^{2}(Q) \leq c|Q| S_{\beta}^{2}(Q) .
\end{aligned}
$$

From this and (4.5)-(4.7) we obtain

$$
\int_{\partial D^{-}} \tilde{u}^{2} d \sigma \leq c|Q| S_{\beta}^{2}(Q)+c|Q|^{1 / 2} S_{\beta}(Q)\left(\int_{\partial D^{-}} \tilde{u}^{2} d \sigma\right)^{1 / 2} .
$$

This yields $\int_{\partial D^{-}} \tilde{u}^{2} d \sigma \leq c|Q| S_{\beta}^{2}(Q)$. Hence, from this and (4.3), (4.4) we get

$$
\inf _{z \in E} N_{\alpha, d_{Q}} \tilde{u}(z) \leq c\left(\left(S_{\beta, h} u\right) \chi_{Q}\right)^{*}((\lambda-\delta)|Q|) .
$$

Choosing $\delta=\lambda / 2$ and taking the supremum over all $E \subset Q$ with $|E|=\lambda|Q|$ completes the proof of Lemma 4.2 
Remark 4.3. It is easy to see that (4.9) implies the inequality

$$
N_{\lambda, \alpha}^{\#} u(x) \leq c S_{\lambda^{\prime} \min \left(\alpha^{n}, 1\right), \beta}^{\#} u(x) \quad\left(0<\lambda^{\prime}<\lambda<1, \alpha<\beta\right),
$$

where $c=c\left(\lambda, \lambda^{\prime}, \alpha, \beta, n\right)$. Hence, for $\alpha \geq 1$ we have the inequality "converse" to (4.1).

\section{Proofs of MAin Results}

Here we always suppose that $x, z \in Q$.

Proof of Theorem 3.1. Let as above $\alpha^{\prime}=\max (1 / \alpha, 1)$. Since

$$
K_{\alpha}(t)=\{y:|y-z|<\alpha t\} \backslash\{y:|y-x|<\alpha t\} \subset\left\{y: \alpha t-d_{Q} \leq|y-z|<\alpha t\right\},
$$

for $t>\alpha^{\prime} d_{Q}$ we get

$$
\left|K_{\alpha}(t)\right| \leq c_{n} n \alpha^{n-1} d_{Q} t^{n-1} .
$$

From this and (2.2) we obtain

$$
\begin{aligned}
\int_{\alpha^{\prime} d_{Q}}^{\infty} & \int_{\{|y-z|<\alpha t\}} t^{1-n}|\nabla u(y, t)|^{2} d y d t \\
\leq & \int_{\alpha^{\prime} d_{Q}}^{\infty} \int_{K \alpha(t)} t^{1-n}|\nabla u(y, t)|^{2} d y d t \\
& +\int_{\alpha^{\prime} d_{Q}}^{\infty} \int_{\{|y-x|<\alpha t\}} t^{1-n}|\nabla u(y, t)|^{2} d y d t \\
\leq & c d_{Q} N_{\beta}^{2} u(z) \int_{\alpha^{\prime} d_{Q}}^{\infty} \frac{d t}{t^{2}}+S_{\alpha}^{2} u(x) \leq c N_{\beta}^{2} u(z)+S_{\alpha}^{2} u(x) .
\end{aligned}
$$

If $\alpha<1$, then using (2.2) again, we get

$$
\int_{d_{Q}}^{\alpha^{\prime} d_{Q}} \int_{\{|y-z|<\alpha t\}} t^{1-n}|\nabla u(y, t)|^{2} d y d t \leq c N_{\beta}^{2} u(z) \int_{d_{Q}}^{\alpha^{\prime} d_{Q}} \frac{d t}{t} \leq c N_{\beta}^{2} u(z) .
$$

Thus, two last estimates show that for any $\alpha>0$ and for all $z \in Q$,

$$
\begin{aligned}
S_{\alpha}^{2} u(z) & =S_{\alpha, d_{Q}}^{2} u(z)+\int_{d_{Q}}^{\infty} \int_{\{|y-z|<\alpha t\}} t^{1-n}|\nabla u(y, t)|^{2} d y d t \\
& \leq S_{\alpha, d_{Q}}^{2} u(z)+c N_{\beta}^{2} u(z)+S_{\alpha}^{2} u(x) .
\end{aligned}
$$

From this and (4.1) we have

$$
\begin{aligned}
\left(\left(S_{\alpha}^{2} u\right) \chi_{Q}\right)^{*}(\lambda|Q|) & \leq\left(\left(S_{\alpha, d_{Q}}^{2} u\right) \chi_{Q}\right)^{*}(\lambda / 2|Q|)+c\left(\left(N_{\beta}^{2} u\right) \chi_{Q}\right)^{*}(\lambda / 2|Q|)+S_{\alpha}^{2} u(x) \\
& \leq c m_{\lambda / 4} N_{\beta}^{2} u(x)+c m_{\lambda / 2} N_{\beta}^{2} u(x)+S_{\alpha}^{2} u(x) \\
& \leq c m_{\lambda / 4} N_{\beta}^{2} u(x)+S_{\alpha}^{2} u(x)
\end{aligned}
$$

(with $\lambda^{\prime}=\lambda / 2$ in (4.1)). Taking the upper bound over all $Q \ni x$ completes the proof of the theorem. 
Proof of Theorem [3.2. We use here the notation from Lemma 4.2. Let $(y, t) \in$ $\Gamma_{\alpha}(z)$. If $t \geq d_{Q}$, then $\left(x, \alpha^{\prime} t\right) \in \Gamma_{\alpha}(z)$. Using Mean Value Theorem and (2.3), we obtain

$$
|u(y, t)| \leq\left|u(y, t)-u\left(x, \alpha^{\prime} t\right)\right|+N^{+} u(x) \leq c S_{\beta} u(z)+N^{+} u(x) .
$$

Hence,

$$
\begin{aligned}
N_{\alpha} u(z) & =\max \left(\sup _{(y, t) \in \Gamma_{\alpha, d_{Q}}(z)}|u(y, t)|, \sup _{(y, t) \in \Gamma_{\alpha} \backslash \Gamma_{\alpha, d_{Q}}(z)}|u(y, t)|\right) \\
& \leq \max \left(N_{\alpha, d_{Q}} \tilde{u}(z)+N^{+} u(x), c S_{\beta} u(z)+N^{+} u(x)\right) \\
& \leq N_{\alpha, d_{Q}} \tilde{u}(z)+c S_{\beta} u(z)+N^{+} u(x) .
\end{aligned}
$$

From this and Lemma 4.2 we get

$$
\begin{aligned}
\left(\left(N_{\alpha} u\right) \chi_{Q}\right)^{*}(\lambda|Q|) & \leq\left(\left(N_{\alpha, d_{Q}} \tilde{u}\right) \chi_{Q}\right)^{*}(\lambda|Q| / 2)+c\left(\left(S_{\beta} u\right) \chi_{Q}\right)^{*}(\lambda|Q| / 2)+N^{+} u(x) \\
& \leq c m_{\lambda / 4} S_{\beta} u(x)+c m_{\lambda / 2} S_{\beta} u(x)+N^{+} u(x) \\
& \leq c m_{\lambda / 4} S_{\beta} u(x)+N^{+} u(x),
\end{aligned}
$$

which implies the desired result.

Proof of Theorem 3.3. It suffices to prove the theorem for $0<\alpha<1$. Let $\eta<\alpha$.

If $t>d_{Q} / \eta$, then $(x, t) \in \Gamma_{\eta}(z)$. Using Mean Value Theorem and (2.2), we have

$$
\begin{aligned}
\left.|| \nabla u(z, t)\right|^{2}-|\nabla u(x, t)|^{2} \mid & \leq(|\nabla u(z, t)|+|\nabla u(x, t)|)|| \nabla u(z, t)|-| \nabla u(x, t)|| \\
& \leq c \frac{|z-x|}{t} N_{\alpha} u(z)|\nabla| \nabla u(\xi, t)|| \leq c \frac{d_{Q}}{t^{3}} N_{\alpha}^{2} u(z),
\end{aligned}
$$

where $(\xi, t)$ is a point on the line with the end points $(z, t)$ and $(x, t)$. From this and (2.2) we obtain

$$
\begin{aligned}
& \int_{d_{Q}}^{\infty} t|\nabla u(z, t)|^{2} d t=\int_{d_{Q}}^{d_{Q} / \eta} t|\nabla u(z, t)|^{2} d t+\int_{d_{Q} / \eta}^{\infty} t|\nabla u(z, t)|^{2} d t \\
& \leq c N_{\alpha}^{2} u(z) \int_{d_{Q}}^{d_{Q} / \eta} \frac{d t}{t}+c d_{Q} N_{\alpha}^{2} u(z) \int_{d_{Q}}^{\infty} \frac{d t}{t^{2}}+\int_{d_{Q}}^{\infty} t|\nabla u(x, t)|^{2} d t \\
& \leq c N_{\alpha}^{2} u(z)+g^{2} u(x) .
\end{aligned}
$$

Next, we note that besides the well-known inequality $g(x) \leq c S(x)$ (see [12]), one can prove its "truncated" variant using exactly the same argument:

$$
\int_{0}^{d_{Q}} t|\nabla u(z, t)|^{2} d t \leq c_{\eta, n} S_{\eta, d_{Q}}^{2} u(z) .
$$

Thus, applying Lemma 4.1 and (5.1), we get

$$
\begin{aligned}
\left(\left(g^{2} u\right) \chi_{Q}\right)^{*}(\lambda|Q|) & \leq c\left(S_{\lambda / 2, \eta}^{\#} u(x)\right)^{2}+c m_{\lambda / 2} N_{\alpha}^{2} u(x)+g^{2} u(x) \\
& \leq c m_{\lambda / 4}\left(N_{\alpha}^{2} u\right)(x)+g^{2} u(x),
\end{aligned}
$$

as required.

Proof of Theorem 3.4. Note, that in [10] the "good- $\lambda$ " inequality relating $g_{\mu}$ and $T_{\mu}$ have been proved. Using essentially the same arguments, one can prove the desired pointwise estimate. So, we shall omit some details in the proof. 
We shall use the following inequalities [10], 12, p. 275]:

$$
\begin{gathered}
N_{\alpha} u(x) \leq c T_{\mu} u(x), \\
|\nabla u(y, t)|^{2} \leq c t^{-2} \frac{1}{t^{n+1}} \int_{B_{t / 2}(y, t)} u^{2}(\xi, \eta) d \xi d \eta,
\end{gathered}
$$

where $B_{t / 2}(y, t)$ is the ball centered at $(y, t)$ of radius $t / 2$.

We split the integral defining $g_{\mu}$ into two as follows:

$$
\begin{aligned}
g_{\mu}^{2}(u)(z) & =\int_{T(3 Q)}\left(\frac{t}{t+|z-y|}\right)^{\mu n} t^{1-n}|\nabla u(y, t)|^{2} d y d t \\
& +\int_{\mathbb{R}_{+}^{n+1} \backslash T(3 Q)}\left(\frac{t}{t+|z-y|}\right)^{\mu n} t^{1-n}|\nabla u(y, t)|^{2} d y d t \\
& \equiv g_{\mu, 1} u(z)+g_{\mu, 2} u(z) .
\end{aligned}
$$

Using (5.3) and the standard techniques for maximal functions, one can show that

$$
g_{\mu, 2} u(z) \leq c T_{\mu}^{2} u(z)+g_{\mu}^{2}(x) .
$$

Now we set $A=\left\{z \in Q: T_{\mu} u(z) \leq\left(\left(T_{\mu} u\right) \chi_{Q}\right)^{*}(\lambda|Q| / 2)\right\}$. Let $E \subset Q,|E|=\lambda|Q|$.

Then $|E \cap A| \geq \lambda|Q| / 2$. Denote $D=\bigcup_{z \in A} \Gamma_{1,3 d_{Q}}(z)$. We have

$$
\begin{aligned}
\inf _{z \in E} g_{\mu, 1} u(z) & \leq \frac{1}{|E \cap A|} \int_{E \cap A} \int_{T(3 Q)}\left(\frac{t}{t+|z-y|}\right)^{\mu n} t^{1-n}|\nabla u(y, t)|^{2} d y d t d z \\
& \leq \frac{c}{|Q|} \int_{T(3 Q) \cap D} \int_{A}\left(\frac{t}{t+|z-y|}\right)^{\mu n} t^{1-n}|\nabla u(y, t)|^{2} d z d y d t \\
& +\frac{c}{|Q|} \int_{T(3 Q) \backslash D} \int_{A}\left(\frac{t}{t+|z-y|}\right)^{\mu n} t^{1-n}|\nabla u(y, t)|^{2} d z d y d t .
\end{aligned}
$$

Since $\int_{\mathbb{R}^{n}} \frac{t^{(\mu-1) n}}{(t+|z-y|)^{\mu n}} d z \leq c_{\mu, n}$, we see that

$$
\int_{T(3 Q) \cap D} \int_{A}\left(\frac{t}{t+|z-y|}\right)^{\mu n} t^{1-n}|\nabla u(y, t)|^{2} d z d y d t \leq c \int_{D} t|\nabla u(y, t)|^{2} d y d t .
$$

Using (5.2) and the same arguments as in Lemma 4.1 we obtain

$$
\begin{aligned}
\int_{D} t|\nabla u(y, t)|^{2} d y d t & \leq c|Q| \sup _{z \in A} N_{\alpha}^{2} u(z) \\
& \leq c|Q| \sup _{z \in A} T_{\mu}^{2} u(z) \leq c|Q|\left(\left(T_{\mu}^{2} u\right) \chi_{Q}\right)^{*}(\lambda|Q| / 2) .
\end{aligned}
$$

The last integral in (5.4) is estimated exactly as in [10], by applying Whitney's decomposition to the set $3 Q \backslash A$. Then, using (5.3), one can show that

$$
\int_{T(3 Q) \backslash D} \int_{A}\left(\frac{t}{t+|z-y|}\right)^{\mu n} t^{1-n}|\nabla u(y, t)|^{2} d z d y d t \leq c|Q|\left(\left(T_{\mu}^{2} u\right) \chi_{Q}\right)^{*}(\lambda|Q| / 2) .
$$

Combining the obtained estimates gives

$$
\left(\left(g_{\mu, 1} u\right) \chi_{Q}\right)^{*}(\lambda|Q|) \leq c\left(\left(T_{\mu}^{2} u\right) \chi_{Q}\right)^{*}(\lambda|Q| / 2) .
$$


Hence,

$$
\begin{aligned}
\left(\left(g_{\mu}^{2} u\right) \chi_{Q}\right)^{*}(\lambda|Q|) & \leq c\left(\left(T_{\mu}^{2} u\right) \chi_{Q}\right)^{*}(\lambda|Q| / 4)+c\left(\left(T_{\mu}^{2} u\right) \chi_{Q}\right)^{*}(\lambda|Q| / 2)+g_{\mu}^{2} u(x) \\
& \leq c\left(\left(T_{\mu}^{2} u\right) \chi_{Q}\right)^{*}(\lambda|Q| / 4)+g_{\mu}^{2} u(x),
\end{aligned}
$$

which proves the theorem.

\section{ACKNOWLEDGEMENT}

The author thanks E. Liflyand for numerous stimulating discussions.

\section{REFERENCES}

[1] D.L. Burkholder and R.F. Gundy, Distribution function inequalities for the area integral, Studia Math. 44 (1972), 527-544. MR 49:5309

[2] R.R. Coifman and C. Fefferman, Weighted norm inequalities for maximal functions and singular integrals, Studia Math. 15 (1974), 241-250. MR 50:10670

[3] R.R. Coifman and R. Rochberg, Another characterization of BMO, Proc. Amer. Math. Soc. 79 (1980), 249-254. MR 81b:42067

[4] C. Fefferman and E.M. Stein, $H^{p}$ spaces of several variables, Acta Math. 129 (1972), 137-193. MR 56:6263

[5] R. Fefferman, R.F. Gundy, M. Silverstein and E.M. Stein, Inequalities for ratios of functionals of harmonic functions, Proc. Nat. Acad. Sci. U.S.A. 79 (1982), 7958-7960. MR 85c:42024

[6] M. de Guzman, Differentiation of integrals in $\mathbb{R}^{n}$, Lecture Notes in Math., 481, SpringerVerlag 1975. MR 56:15866

[7] D.S. Kurtz, Rearrangement inequalities for Littlewood-Paley operators, Math. Nachr. 133 (1987), 71-90. MR 89c:42016

[8] M.A. Leckband, A note on exponential integrability and pointwise estimates of LittlewoodPaley functions, Proc. Amer. Math. Soc. 109 (1990), 185-194. MR 91f:42020

[9] A.K. Lerner, On pointwise estimates for maximal and singular integral operators, Studia Math. 138 (2000), 285-291. MR 2001b:42016

[10] B. Muckenhoupt and R.L. Wheeden, Norm inequalities for the Littlewood-Paley function $g_{\lambda}^{*}$, Trans. Amer. Math. Soc. 191 (1974), 95-111. MR 52:8810

[11] T. Murai and A. Uchiyama, Good $\lambda$ inequalities for the area integral and the nontangential maximal function, Studia Math. 83 (1986), 251-262. MR 87m:42016]

[12] E.M. Stein, Singular Integrals and differentiability properties of functions, Princeton Univ. Press 1970. MR 44:7280

[13] J.-O. Strömberg, Bounded mean oscillation with Orlicz norms and duality of Hardy spaces, Indiana Univ. Math. J. 28 (1979), 511-544. MR 81f:42021

Department of Mathematics and Computer Science, Bar-Ilan University, 52900 RaMAT GAN, ISRAEL

E-mail address: aklerner@netvision.net.il 